\title{
The effect of cooling and reheating sweet potato (ipomoea batatas) on postprandial glycaemic response in females
}

\author{
C. Foster-Brown ${ }^{1}$ and Dr. A. Majumdar ${ }^{1}$ \\ ${ }^{1}$ School of Health and Social Sciences, St. Mary's University, Twickenham, TW1 4SX
}

Chronic disease arising from postprandial hyperglycaemia and insulinaemia is epidemic in the UK. Rapidly digested carbohydrates are largely responsible for elevated postprandial glycaemic-response (PGR); however, carbohydrates containing resistant starch (RS) reduce post-prandial hyperglycaemia/ insulinaemia ${ }^{(1-2)}$. Research shows carbohydrate preparation and processing techniques results in starch retrogradation, increasing resistant starch content ${ }^{(3)}$. This study's main aim was to determine the effect cooking, cooling, and reheating sweet potato (RSP) had on RS content by analysing PGR in female participants. Further aims were to investigate whether RSP increased satiety compared to fresh sweet potato (FSP), to establish if significant correlations existed between PGR and participant characteristics, and to explore whether participant menstruation cycles affected PGR to test carbohydrates.

The study was a controlled single-blind (participant was blinded) clinical trial. Ten female participants (20-48years) were recruited to attend 5 testing sessions. At each session fasted participants consumed $50 \mathrm{~g}$ of carbohydrate; dextrose on the first 3 , followed by boiled FSP, and boiled, cooled $(24$ hours $(\leq 4 \mathrm{C}))$ and RSP. On each occasion fasting plasma glucose was measured at -5 and 0 mins, and postprandially at 15,30,45,60,90, and 120mins to determine PGR. Hunger Visual Analogue Scales (VAS) were completed at 0,15,30,45,60,90, and 120mins. Participants completed questionnaires relating to medical/ physical activity histories, smoking habits, food allergies, menstrual cycles, and reproductive history.

Results: No statistically significant data was established (p-values $<0.05$ were significant). However, visual non-significant variation in PGR between FSP and RSP is visible in figure 1. No significant correlations were found between PGR and participants characteristics. As participants were tested independent of menstrual cycles, no significant findings were established for whether participants menstrual cycles affected PGR to test carbohydrates (dextrose, FSP, and RSP). Discussion: Research shows the longer and colder starch is cooled for $(\leq 4 \mathrm{C})$, the higher amount of RS3 is formed. This study did not identify variations of satiety based on FSP versus RSP status; this may have resulted from appetite variations affected by menstrual fluctuations of oestrogen levels. No significant correlations were found between PGR and participants characteristics. Participants were tested independent of menstrual cycle status, and no significant findings were established for whether participant menstrual cycles affected PGR to test carbohydrates (dextrose, FSP, and RSP).

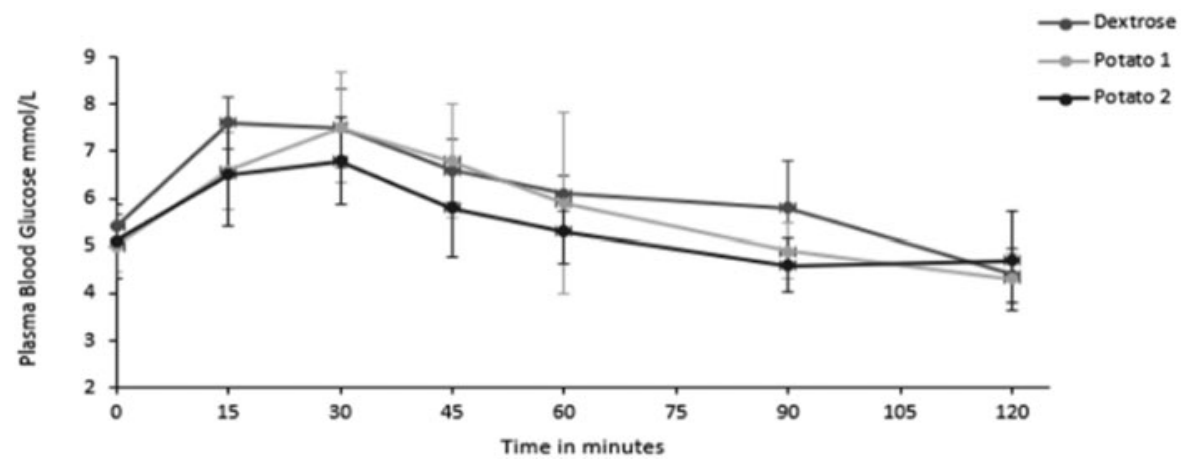

Fig. 1. PGR for participants for all three carbohydrates. Error bars represent $\pm 1 \mathrm{SD}$.

Although non-significant, the visible difference in PGR between RSP and FSP may warrant further research with larger sample sizes and oestrogen status measurement to further explore the issue of RS, PGR and menstrual status.

1. Wong THT \& Louie JCY. Starch. 2017;69:1600205-14.

2. Bird AR Conlon MA, et al. Benef Microbes. 2010;1(4):423-31.

3. Ratnayake WS \& Jackson DS. Adv Food Nutr Res. 2009;55:221-68. 